\title{
Thermosensitive Splicing of a Clock Gene and Seasonal Adaptation
}

\author{
W.-F. CHen, K.H. LOW, C. LIM, AND I. EDERY \\ Department of Molecular Biology and Biochemistry, Rutgers University, Center for Advanced \\ Biotechnology and Medicine, Piscataway, New Jersey 08854
}

\begin{abstract}
Similar to many diurnal animals, the daily distribution of activity in Drosophila exhibits a bimodal pattern with clock-controlled morning and evening peaks separated by a midday "siesta." In prior work, we showed that the thermosensitive splicing of a 3' terminal intron in the RNA product from the Drosophila period (per) gene (dper) is critical for temperature-induced adjustments in the timing of evening activity. Cold temperatures enhance the splicing efficiency of this intron (termed dmpi8, Drosophila melanogaster per intron 8), an event that stimulates the daily accumulation of $d p e r$ RNA and protein, leading to earlier evening activity. Conversely, warm temperatures attenuate dmpi8 splicing efficiency contributing to delayed evening activity, likely ensuring that flies avoid activity during the hot midday sun when they are at increased risk of desiccation. Here, we discuss the underlying molecular mechanisms governing the thermosensitive splicing of dmpi8 and how it contributes to seasonal changes in the daily activity patterns of Drosophila. On a broader perspective, RNA-RNA interactions likely have fundamental roles in the thermal adaptation of life forms to the daily and seasonal changes in temperature.
\end{abstract}

\section{INTRODUCTION}

Organisms on this planet have adapted to the daily rotation of the earth on its axis. By means of endogenous circadian ( $\cong 24$ hours) "clocks" or pacemakers that can be synchronized to the daily and seasonal changes in external time cues, most notably, visible light and ambient temperature, life forms anticipate environmental transitions, perform activities at biologically advantageous times during the day, and undergo characteristic seasonal responses (for review, see Hastings et al. 1991; Edery 2000). Light is almost certainly the predominant entraining agent in nature. Under natural conditions the light/dark (LD) cycle aligns the phases of clocks and evokes daily adjustments in the approximately 24-hour endogenous periods of these oscillators such that they precisely match the 24-hour solar day. The duration of day length (photoperiod) can modify the temporal alignment between a circadian rhythm and local time. A physiologically relevant advantage of this inherent flexibility of clocks is that the daily distributions of physiological and behavioral rhythms are not rigidly locked to local time but can be adjusted for seasonal changes in day length. Despite the obvious importance of photoperiod, ambient temperature is also a key environmental modality regulating the timing of circadian rhythms (Sweeney and Hastings 1961; Rensing and Ruoff 2002). This makes intuitive sense because in temperate latitudes, seasonal changes in day length are also accompanied by predictable changes in average daily temperatures. The work presented here is mainly focused on using Drosophila as a model organism to address how a circadian system contributes to temperature-dependent changes in the distribution of daily activity.

Before discussing this work, however, it is important to recognize that temperature has multiple effects on circadian-regulated phenomena (Rensing and Ruoff 2002). Perhaps the best known but least understood response of circadian rhythms to temperature is that period length is relatively constant over a wide range of physiologically relevant temperatures, an active mechanism termed "temperature compensation." The ability of circadian timing systems to maintain stable periods despite changes in temperature is important because whether it is summer or winter, the solar day does not change in duration. Although period length is rather insensitive to temperature fluctuations, the phases and amplitudes of circadian rhythms can exhibit clear temperature-induced changes. Most notably, circadian rhythms can be phase-shifted by changes in temperature (pulses and step-up or -down) and entrained by daily temperature cycles (see, e.g., Sweeney and Hastings 1961; Wheeler et al. 1993; Liu et al. 1997, 1998; Sidote et al. 1998; and references therein). Other work indicates that the amplitude of a circadian oscillator changes as a function of temperature (usually perceived as increasing with rising temperature), suggested by some as a solution to temperature compensation of period length (LakinThomas et al. 1991; Pittendrigh et al. 1991). In addition, clocks only function within a restricted temperature range, and outside these limits, the oscillatory mechanism appears to be held constant or "stops" (Liu et al. 1997; Revel et al. 2007; and references therein), which might be an extreme example of amplitude reduction.

A less-studied effect of temperature on clock function is based on the observation that the steady-state phases of behavioral rhythms can vary as a function of temperature, even during entrainment by daily light/dark cycles (Sweeney and Hastings 1961). Many animals display a bimodal distribution of activity, with peaks centered around the morning and evening hours that are separated by a midday "siesta" time of varying lengths. A classic example of how temperature affects daily activity patterns is that of garter snakes which are mainly nocturnal at warm temperatures and diurnal at cold temperatures and display a bimodal distribution of activity at intermediate tempera- 
tures (Heckrotte 1961). In general, diurnal animals respond to colder temperatures by displaying a greater proportion of their activity during daytime hours, whereas nighttime activity predominates at warmer temperatures. This directional response has a clear adaptive value, ensuring that the activity of an organism is maximal at a time of day when the temperature would be expected to be optimal for activity (Sweeney and Hastings 1961).

If there is such clear adaptive value, why is this effect of temperature on circadian rhythms less studied? There are several reasons, which also highlight some of the challenges in trying to understand the underlying mechanism and the choices of model systems. First, from a historical perspective, the entrée was based on observing how temperature affects the daily distribution of activity (i.e., movement such as locomotion), which naturally focused the work on animals. Second, it was thought that much of the thermal effect was likely due to the "direct" (or masking) effects of temperature on activity (see, e.g., Matsumoto et al. 1998), minimizing the notion of potential clock involvement. Finally, when it comes to trying to understand "seasonal" adaptation of clocks, much of the focus has been placed on the impact of changing day length or photoperiod. This is quite natural as light is the predominant daily synchronizer in nature, and it is almost certain that circadian rhythms in all organisms, even homeotherms, exhibit phase adjustments in response to changes in day length. Indeed, the dual oscillator model based on coupled morning (M) and evening (E) pacemakers was largely developed over the years as a framework to explain how the $\mathrm{M}$ and $\mathrm{E}$ peaks track sunrise and sunset, respectively, despite seasonal changes in day length (Pittendrigh and Daan 1976). This photocentric view of seasonal adaptation has for the most part not incorporated the effects of temperature on modulating clock dynamics.

Below, we briefly describe some of our work using Drosophila to explore the role of a circadian clock in the adaptation of daily activity patterns to seasonal changes in temperatures. Thermal-sensitive splicing of a clock mRNA has a key role in this adaptation (Majercak et al. 1999). Remarkably, a similar mechanism has also been shown to underlie some temperature responses in the Neurospora clock (Colot et al. 2005; Diernfellner et al. 2005; Brunner and Diernfellner 2006). On a broader perspective, the results suggest that naturally selected variations in the strengths of RNA-RNA interactions offer a simple basis for designing an adaptive molecular mechanism displaying highly calibrated thermal responses that can subsequently impact a wide array of physiological and behavioral programs.

\section{DROSOPHILA AS A MODEL SYSTEM TO STUDY THE ROLE OF CIRCADIAN CLOCKS IN REGULATING THE DAILY DISTRIBUTION OF ACTIVITY AS A FUNCTION OF SEASONAL CHANGES IN TEMPERATURE}

Several years ago, we used D. melanogaster as a model system to understand the role of a clock in regulating the pattern of daily activity as a function of changes in temperature and day length (Majercak et al. 1999). Under stan- dard daily cycles of 12-hour light followed by 12-hour dark (12:12 L:D) at $25^{\circ} \mathrm{C}$, D. melanogaster exhibit a bimodal activity pattern with clock-controlled peaks centered around the lights-on ("morning" peak) and lights-off ("evening" peak) transitions that are separated by a midday dip or "siesta" (Fig. 1) (see, e.g., Hamblen-Coyle et al. 1992). For the purposes of this discussion, it is important to note at the outset that despite the clock-controlled bimodal activity pattern, the evening activity component is largely assayed as a bona fide readout of the circadian system in Drosophila. This is mainly because the morning peak usually coincides with a direct stimulatory effect of light following dawn, and the evening peak most visibly persists in constant darkness (Wheeler et al. 1993). The photoperiod and ambient temperature have effects on the distribution of activity throughout a daily cycle. Over a wide range of photoperiods, the evening peak occurs around the light-todark transition (Qiu and Hardin 1996; Majercak et al. 1999; Rieger et al. 2003; Shafer et al. 2004). As a result, lengthening the photoperiod delays the evening activity relative to the most recent sunrise. Temperature appears to be the main environmental cue determining whether the evening activity will be mostly diurnal or nocturnal (Majercak et al. 1999). With increasing temperature, the evening activity becomes progressively more nocturnal and midday inactivity more pronounced (see Figs. 1 and 3) (Matsumoto et al. 1998; Majercak et al. 1999; Yoshii et al. 2002). This directional response is observed in many diurnal animals, displaying a greater proportion of their activity during the cooler nighttime hours on hot days and conversely the warmer daytime hours during cold days (Sweeney and Hastings 1961).

\section{A Role for Thermal-sensitive Splicing of a Clock Gene in Seasonal Adaptation}

We showed that splicing of the 3 -terminal intron in dper RNA (termed dmpi8) (Cheng et al. 1998) is an important aspect of how the Drosophila circadian clock adapts to seasonal changes in temperature (Majercak et al. 1999). At cold temperatures, the proportion of dmpi8 spliced dper mRNA (termed type $\mathrm{B}^{\prime}$ ) compared to the unspliced variant (termed type A) is enhanced, leading to more rapid daily increases in dper transcript levels and earlier evening activity. Splicing per se, as opposed to retention or removal of the dmpi8 intron, stimulates increases in $d p e r$ mRNA levels. This led to the hypothesis that assembly of active spliceosomes at the 3 '-terminal intron somehow produces more mature transcripts, possibly by facilitating 3 '-end formation (Fig. 1). Transgenic flies bearing variant dper transgenes where splicing of dmpi8 was abrogated manifested nocturnal evening activity even on cold days (Majercak et al. 1999). Long photoperiods partially counteract cold-induced phase advances in the accumulation of dper mRNA and protein by delaying the upswing in TIMELESS (TIM) levels. The functional interrelationships between dPER and TIM ensure that information concerning ambient temperature and light is properly integrated, resulting in activity rhythms that are optimally aligned with the prevailing environmental conditions.

In more recent findings, we and other investigators 


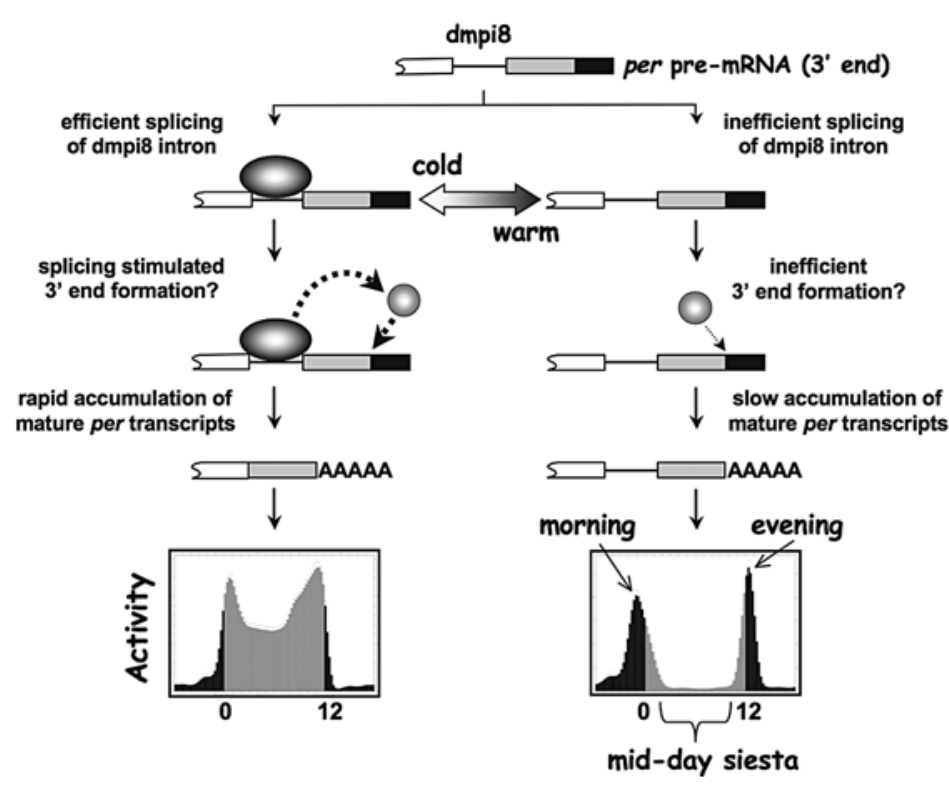

Figure 1. Model for how thermosensitive splicing of the dper 3 '-terminal intron (dmpi8) regulates seasonal changes in the daily distribution of activity in Drosophila melanogaster. Shown at the top is the $3^{\prime}$ end of the dper precursor mRNA (premRNA); (white box) Sequences from translation stop codon to just upstream of $5^{\prime}$ splice site of dmpi8; (horizontal line) dmpi8 intron; (gray box) sequences following 3' splice site of dmpi8 until 3' cleavage and polyadenylation site; (black box) transcribed sequences downstream from 3' cleavage and polyadenylation site. Cold temperatures enhance binding of spliceosome (large oval), which stimulates binding of 3 '-end formation factors (small circle). More rapid accumulation of dper transcripts leads to advanced evening activity and less prominent midday inactivity. Conversely, on warm days, the inefficient splicing of dmpi8 leads to an extended midday siesta and preferential nocturnal evening activity, events that enhance the ability of Drosophila to avoid the deleterious effects associated with the hot midday sun. For Drosophila activity profiles: (black bars) dark; (gray bars) light. Locomotor activity was collected in $15 \mathrm{~min}$ bins and relative values throughout a daily cycle (example shown, $12 \mathrm{hr}$ light:12 hr dark) shown in arbitrary units. (Left panel) $18^{\circ} \mathrm{C}$; (right panel) $29^{\circ} \mathrm{C}$ (also shown are morning and evening activity components, including midday siesta). (Adapted from Majercak et al. 1999.) showed that daily fluctuations in the splicing efficiency of dmpi8 are regulated by the clock in a manner that depends on the photoperiod (day length) and temperature (Collins et al. 2004; Majercak et al. 2004). Shortening the photoperiod enhances dmpi8 splicing and advances its cycle, whereas the amplitude of the clock-regulated daytime decline in splicing increases as temperatures rise. This suggests that at elevated temperatures, the clock has a more pronounced role in maintaining low splicing during the day, an adaptive response that for small insects likely minimizes the risks of desiccation during the hot midday hours. Indeed, on entering direct sunlight, a 10-mg insect can heat up by about $10^{\circ} \mathrm{C}$ in less than 10 seconds (Sayeed and Benzer 1996), a potentially life-threatening event for Drosophila that only weigh in the vicinity of $1 \mathrm{mg}$. In summary, the splicing efficiency of dmpi8 is regulated by the clock, temperature, and day length, ultimately modulating the trajectory of daytime increases in dper mRNA levels and hence clock phase. We propose that the multimodal regulation in the splicing efficiency of dmpi8 acts as a "seasonal sensor" conveying calendar information to the animal. We and others also identified a novel nonphotic role for NORPA (no receptor potential $A$ ) in the regulation of dmpi8 splicing (Collins et al. 2004; Majercak et al. 2004). In this chapter, we do not discuss the role of norp $A$ further, but we focus on the basis for temperature-dependent changes in dmpi8 splicing efficiency.

\section{Weak 5' and 3' Splice Sites as a Basis for Thermosensitive Splicing}

As an initial attempt to understand the basis for the temperature-dependent splicing of dmpi8, we sought to develop a simplified cell-culture-based assay that recapitulates this thermosensitivity phenotype. D. melanogaster (Oregon-R strain [OR]) dper genomic sequences encompassing the entire 3 '-untranslated region (3'UTR) followed by $90 \mathrm{bp}$ of 3 '-flanking nontranscribed region were fused downstream from a luciferase (luc) reporter gene. Expression of the luc-dper/3'UTR hybrid gene was placed under the control of the constitutive actin 5C promoter (pAct) (Fig. 2A) (Angelichio et al. 1991). To enable the simple introduction of different intron and flanking exon sequences, this parent wild-type vector (termed dmpi8) also includes two engineered Xhol and Kpn1 restriction sites placed 10 bp upstream and downstream from the dmpi8 $5^{\prime}$ and $3^{\prime}$ splice sites (ss), respectively. Following transfection of the popular Drosophila Schneider S2 cells, at least two independent lines of stable transformants were isolated for each construct. Subsequently, separate aliquots of cells were incubated for 24 hours at different temperatures in the dark. Total RNA was extracted, and the relative levels of spliced and nonspliced products were determined as previously described using reverse transcriptase-polymerase chain reaction (RT-PCR) whereby values are normalized to an internal RNA standard (Majercak et al. 2004). The dmpi8 control RNA exhibited approximately 2.5-3.2-fold increases $(n>10 ; P<0.001$ using Student's $t$-test for values obtained at $12^{\circ} \mathrm{C}$ and $24^{\circ} \mathrm{C}$ ) in the proportion of spliced-to-unspliced RNA at $12^{\circ} \mathrm{C}$ compared to $24^{\circ} \mathrm{C}$ (Fig. 2D). This is similar to the 2-3-fold increase in the ratio of spliced type $\mathrm{B}^{\prime}$ to unspliced type-A dper RNA variants observed in wild-type flies at $18^{\circ} \mathrm{C}$ compared to $29^{\circ} \mathrm{C}$ (Majercak et al. 1999). Thus, the dper $3^{\prime} \mathrm{UTR}$ is sufficient to manifest thermosensitive splicing of dmpi8 in a 

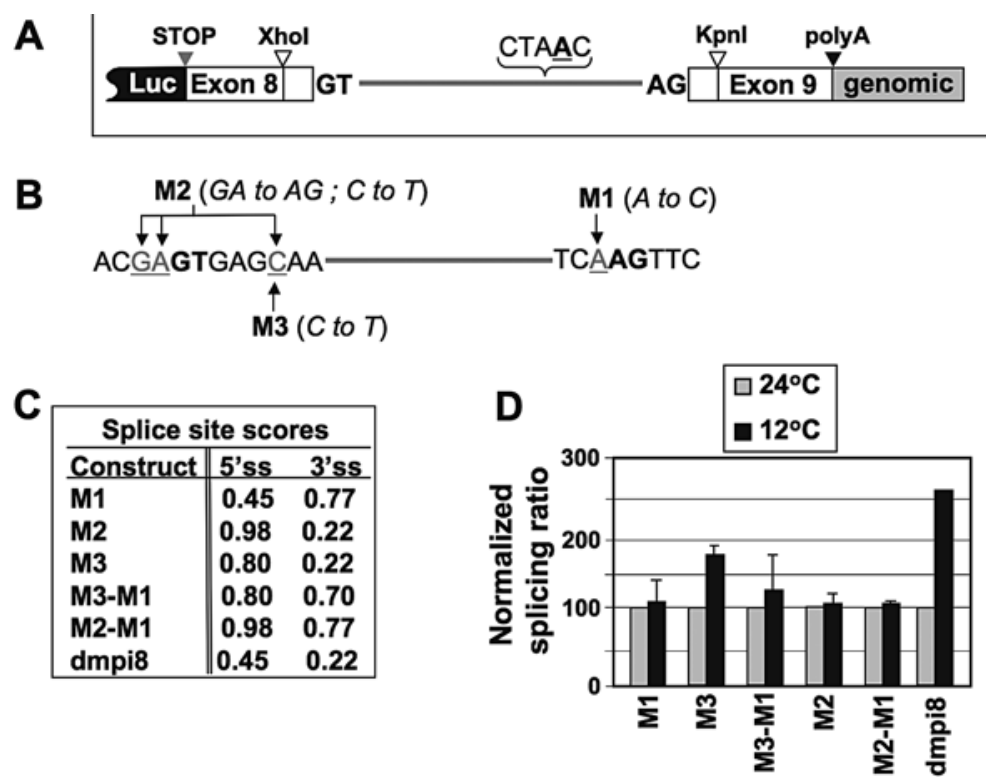

Figure 2. Multiple suboptimal splice sites underlie temperature-dependent splicing of the dmpi8 intron. (A) A luciferase (luc) reporter gene fused to 3' dper sequences was used to transfect S2 cells. Xho1 and Kpn1 sites were engineered into the fusion construct such that they flanked the $5^{\prime}$ and $3^{\prime}$ splice sites of dmpi8 to facilitate swapping intronic sequences. $(B)$ Schematic of mutations made to increase the predicted strengths of the $5^{\prime}$ and/or $3^{\prime}$ splice sites of dmpi8. (C) Predicted scores for the $5^{\prime}$ and $3^{\prime}$ splice sites of the different constructs (range, $0-1$, where 1 is the highest). $(D)$ Cells were grown at either $12^{\circ} \mathrm{C}$ or $24^{\circ} \mathrm{C}$, and the relative splicing efficiency was determined (values for $24^{\circ} \mathrm{C}$ were set to 1 and the rest of the values normalized). Note that whereas dmpi8 exhibits approximately 2.5-fold more efficient splicing at $12^{\circ} \mathrm{C}$ compared to $24^{\circ} \mathrm{C}$, the other transcripts exhibit little (e.g., M3) to no (e.g., M2) temperaturesensitive splicing.

simplified cell culture system, providing a powerful approach to investigate mechanistic issues.

A feature of the dper 3 -terminal intron in $D$. melanogaster that caught our attention was that both the $5^{\prime}$ and $3^{\prime}$ splice sites were predicted to be weak. For example, using a program that predicts splice sites in $D$. melanogaster (www.fruitfly.org/seq_tools/splice.html), dmpi8 has the lowest scores for both the $5^{\prime}($ score $=0.45)$ and $3^{\prime}$ ( score $=0.21$ ) splice sites of all the introns in per (the other per small introns had scores of 0.88-0.97 for the $5^{\prime}$ and $0.42-0.95$ for the $3^{\prime}$ splice sites, with 1 being highly likely) (Fig. 2C). The presumptive branchpoint (BP) lies in a sequence that is very similar but not identical to the consensus sequence for D. melanogaster introns (CUAAU) (Fig. 2A). Multiple suboptimal splices sites for the dmpi8 intron was eerily reminiscent of prior work from Murphy and co-workers studying the basis for the thermosensitive splicing phenotype of the ts 110 mutant of Moloney murine sarcoma virus (Mo-MSVts110). A series of elegant studies showed that multiple suboptimal splicing signals were the basis for the temperature-sensitive splicing of Mo-MSVts110 (Cizdziel et al. 1988; Touchman et al. 1995; Ainsworth et al. 1996). For example, introducing a consensus branchpoint signal increased splicing efficiency and abrogated the thermodependency (Touchman et al. 1995). A similar result was observed by strengthening the 5'splice site (Ainsworth et al. 1996). Intriguingly, other random mutagenesis strategies to generate temperature-sensitive phenotypes were eventually shown to be a result of introducing mutations that weaken key splicing signals on precursor mRNAs (pre-mRNAs).
Indeed, increasing the predicted strength of either the $5^{\prime}$ splice site (e.g., M2) or the $3^{\prime}$ splice site (e.g., M1) in dmpi8 eliminated thermosensitivity, whereas in other cases, the thermal response was diminished (e.g., M3) (Fig. 2B-D). Thus, there is a graded response that correlates with the predicted strengths of the $5^{\prime}$ and $3^{\prime}$ signals. As with Mo-MSVts110, our findings suggest that temperature regulation of dmpi8 splicing is not driven by discrete thermosensitive elements but involves an intricate balance of multiple suboptimal splice sites, raising the possibility of intricate coevolution. Studies using transgenic flies whereby we replaced the wild-type $D$. melanogaster 3'UTR (from the standard Oregon-R strain) with the M2 version also exhibited near complete splicing at all test temperatures (data not shown). Moreover, in both $\mathrm{S} 2$ cells and flies, versions of the dper 3 '-terminal intron with higher splicing efficiency also exhibited higher RNA levels (data not shown). Together, these results are highly consistent with our earlier model that the splicing efficiency of the $d$ per 3 '-terminal intron stimulates the production of mature transcripts.

\section{A Similar Mechanism in the Neurospora Clock}

Along with Drosophila, work in Neurospora laid the early foundation for mechanistic insights underlying clock mechanisms (Dunlap 1996). The initial biochemical characterization of the FREQUENCY (FRQ) protein led to a remarkable realization that has grown over the years and can be equally applied to other clock systems. Both dPER and FRQ exhibit dramatic progressive 
increases in phosphorylation throughout a daily cycle that are intimately linked to abundance, whereby the most hyperphosphorylated isoforms are associated with sharp decreases in levels (Edery et al. 1994b; Garceau et al. 1997). Today, we know that despite the lack of sequence conservation, there are uncanny similarities between dPER and FRQ proteins with respect to clock function. Importantly, both have been shown to be bona fide "state variables" in their respective clockworks (Aronson et al. 1994; Edery et al. 1994a), indicating that the daily fluctuations in the abundance/activity of these proteins, as opposed to simply their presence, are inextricably linked to the phase of the clock. dPER and FRQ proteins share similar modes of action, whereby they participate in transcriptional autoinhibitory loops crucial to clock function, most likely by acting as conduits for the timely delivery or regulation of trans-acting factors that more directly inhibit the key transcription factors which drive their own expression (Schafmeier et al. 2005; He et al. 2006; Yu et al. 2006; Kim et al. 2007). Although we have gotten used to the idea by now, it is still rather intriguing that virtually identical kinases, phosphatases, and F-box proteins regulate the daily life cycles of PER and FRQ proteins (Gallego and Virshup 2007).

Perhaps then, it should not be too unexpected that an aspect of $f r q$ expression regulated by temperature involves mRNA splicing. Earlier work from Dunlap and co-workers showed that temperature regulates the relative levels of two isoforms of FRQ protein, a short (s-FRQ) and long (1-FRQ) version that arise from alternative use of translation initiation sites (Liu et al. 1997). More recent work from the Brunner and Dunlap labs demonstrated that the ratio of 1FRQ versus s-FRQ is regulated by thermosensitive splicing of an intron that when excised removes the translation initiation site of 1-FRQ (Colot et al. 2005; Diernfellner et al. 2005; Brunner and Diernfellner 2006). Moreover, inefficient recognition of nonconsensus splice sites at elevated temperatures was shown to underlie the thermosensitivity (Diernfellner et al. 2005; Brunner and Diernfellner 2006). Additional steps including temperature-dependent effects on the rate of ribosome scanning at the different translation initiation recognition sequences allow for adjustments in FRQ levels that are appropriate for the local ambient temperature (Diernfellner et al. 2005; Brunner and Diernfellner 2006). Thus, in two widely different species, the clockworks adapt to changes in temperature by thermal adjustments in the levels of key state variables via a mechanism involving an initial thermosensitive splicing event that has ramifications for other more downstream aspects of mRNA metabolism or utilization, such as the efficiency of 3 '-end formation in the case of dper or translation for frq.

\section{HOW DO WEAK SPLICING SIGNALS YIELD THERMOSENSITIVE SPLICING?}

From the aforementioned examples, such as dper, frq, and Mo-MSVts110, it is clear that the presence of multiple suboptimal splicing signals can render the splicing efficiency of an intron temperature-dependent. In very general terms, pre-mRNA splicing in eukaryotes occurs via two major steps that differ in the need for ATP consumption (for review, see Hastings and Krainer 2001). The first step is an ATP-hydrolysis-independent assembly of the core splicing machinery or spliceosome to an intronic sequence. This involves the recognition of cis-acting signals on the mRNA such as the 5' and 3' splice sites and 3' polypyrimidine tracts. Subsequently, in the second phase, which requires ATP hydrolysis, the pre-mRNA and splicing machinery undergo structural rearrangements, culminating in cleavage at the $5^{\prime}$ intronic site, lariat formation, and finally, removal of the intron with concomitant ligation of the exonic sequences. The initial binding of the spliceosome and the subsequent catalytic events are heavily dependent on dynamic interactions between the substrate transcript and the small nuclear RNAs (snRNAs) that are core constituents of the splicing machinery.

Given the ability of RNA to form intramolecular and intermolecular base-pairing interactions of varying strengths, splicing offers a rich opportunity for generating a molecular mechanism that can elicit calibrated thermal responses. On the basis of the important roles for the $5^{\prime}$ and 3' splice sites in spliceosome docking, it is likely that for introns with multiple suboptimal splicing signals, low temperatures stabilize RNA-RNA and/or RNA-protein interactions between the splicing machinery and the RNA substrate, leading to more efficient splicing. For example, low temperatures could enhance the binding of the U1 snRNA to nonconsensus 5 ' splice sites, modulating a major rate-limiting step in overall splicing efficiency. It is also possible that temperature has effects during the catalytic phase of splicing. For example, the U4 and U6 snRNAs undergo structural rearrangements between them that can be modulated by temperature (Shannon and Guthrie 1991). However, this type of mechanism lacks substrate specificity and would have global effects on splicing. Other possibilities include temperature-dependent changes in (1) RNA secondary structure that modulates the ability of an intron to be recognized and excised and (2) binding of trans-acting regulatory splicing factors, such as SR proteins that can enhance or suppress splicing (Bourgeois et al. 2004).

\section{LIGHT-INDUCTION OF TIM EXPRESSION AT COLD TEMPERATURES: AN INTEGRATED MECHANISM}

Other work from our lab also showed that light stimulates expression of tim at low but not warm temperatures (Chen et al. 2006). Intriguingly, although numerous lines of evidence indicate that dper and tim expression are activated by the same mechanism, light has no measurable acute effect on dper mRNA abundance (Chen et al. 2006). The acute effects of light on tim expression are temporally gated, essentially restricted to the daily rising phase in tim mRNA levels. Because the start of the daily upswing in tim expression begins several hours after dawn in long photoperiods (day length), this gating mechanism likely ensures that sunrise does not prematurely stimulate tim expression during unseasonally cold spring/summer days. We suggest that photic stimulation of tim expression at low temperatures is part of a seasonal adaptive response that helps advance the phase of the clock on cold days, enabling flies to exhibit preferential daytime activity 

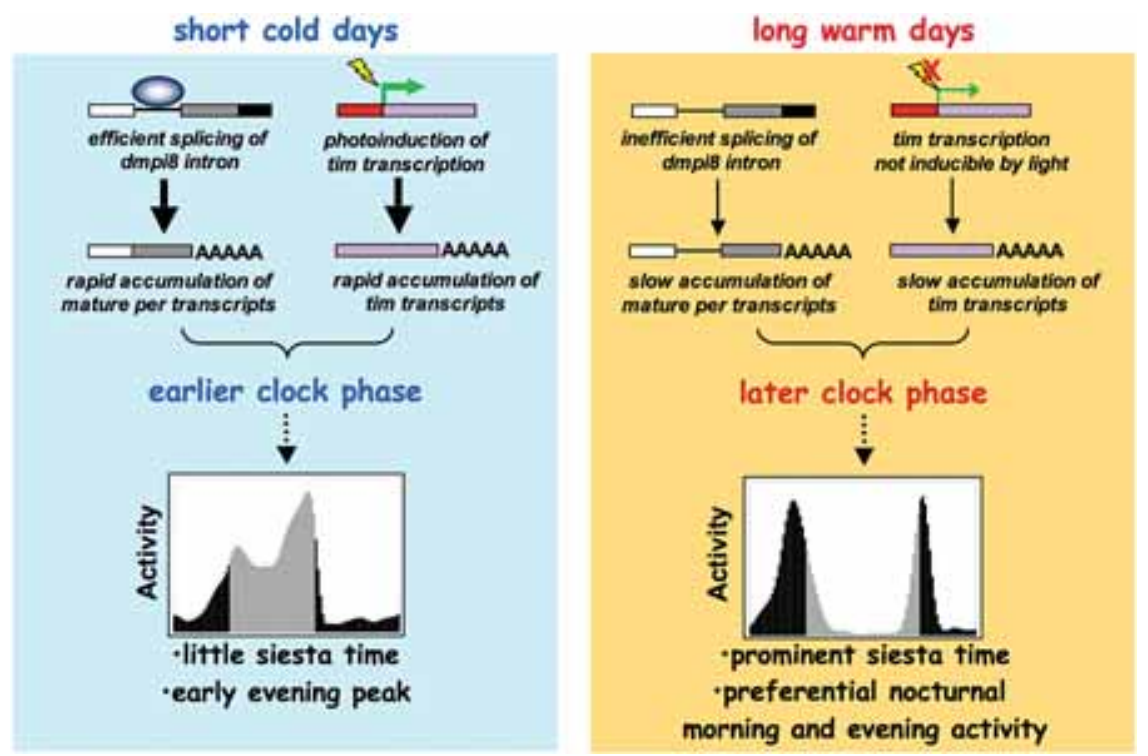

Figure 3. Integrated effects of photoperiod and temperature on dper and tim expression. Cold short days stimulate the rapid accumulation of both dper and tim transcripts, leading to advanced evening activity and little to no midday siesta. Long photoperiods and warm temperatures delay the daily upswings in $d p e r$ and tim transcripts, contributing to preferential nocturnal evening activity and pronounced midday inactivity. Thus, temperature and day length have parallel effects on the daily accumulation profiles in dper and tim transcripts.

despite the (usually) earlier onset of dusk. Thus, we envisage an integrated mechanism whereby during autumn/ winter seasons, splicing of dmpi8 is enhanced, leading to a more rapid rise in dper RNA levels, and light stimulates tim induction, responses that function in a coordinate manner to advance the timing of when de-novo-synthesized PER and TIM proteins begin to interact, leading to an earlier clock phase (Fig. 3). Otherwise stated, the ability of temperature and photoperiod to adjust trajectories in the rising phases of one or more clock RNAs constitutes a major mechanism contributing to seasonal adaptation of clock function in Drosophila.

This raises the role of rhythmic clock gene expression in light of recent findings in cyanobacteria which clearly show that a biochemical oscillator with circadian properties can be reconstituted in vitro (Nakajima et al. 2005). In this case, mixing purified recombinant versions of the key clock proteins $\mathrm{KaiA}, \mathrm{KaiB}$, and $\mathrm{KaiC}$ in the presence of ATP as an energy source resulted in daily cycles in the phosphorylated state of KaiC. This dramatic result convincingly shows that circadian molecular cycles can occur in the absence of cycling gene expression. KaiC could be considered the equivalent of APER and FRQ in that these proteins undergo daily cycles in phosphorylation that are likely to be critical signals in period determination and defining the state of the clock. Although it is possible that reconstituted rhythms in dPER or FRQ phosphorylation might be attained, rhythmic clock gene expression is likely to be an important element in optimizing the dynamics of clock systems.

\section{CONCLUSIONS}

The work described herein focused on the use of $D$. melanogaster to study the role of a circadian system in modulating daily activity patterns in response to seasonal changes in temperature. This species has also contributed to other effects of temperature on circadian systems. For example, the elegant work from Kyriacou and colleagues showed that a stretch of Thr-Gly (TG) repeats centrally located in the dPER-coding region modulates temperature compensation of period length (Sawyer et al. 1997). Importantly, these TG repeats are subject to natural selection and exhibit a latitudinal cline in natural populations of D. melanogaster (Sawyer et al. 1997). Thus, dPER activity appears to be a focal point for thermal responses in the Drosophila clock. Ongoing studies are aimed at determining whether natural selection is also operating at the level of dmpi8 splicing efficiency and whether there is an association between the thermosensitivty of 3 '-terminal splicing and the type of TG repeat present on $d p e r$ (i.e., diurnal phase adjustments can also be attained by changes in period length). More recent work has also identified a factor termed nocte that specifically modulates circadian entrainment to daily changes in temperature but not light/dark cycles (Glaser and Stanewsky 2005). Intriguingly, nocte appears to be unrelated to dmpi8 splicing, indicating that multiple pathways convey thermal information to the circadian system in flies.

Among the most exciting recent findings in the study of circadian rhythms was the identification of long-sought "morning" and "evening" pacemakers in D. melanogaster. The $M$ cells preferentially regulate the morning peak of activity, whereas the E cells drive the evening component (Grima et al. 2004; Stoleru et al. 2004). Storelu, Rosbash, and coworkers showed that day length can modulate the relative impact of either $\mathrm{M}$ and $\mathrm{E}$ cells on the overall circadian system (Stoleru et al. 2007). For example, during long nights, the $\mathrm{M}$ cells dominate, whereas during long days, the E cells are predominant. A current challenge is to 
integrate the photoperiodic effects on M-E pacemakers with temperature. In this regard, a curious feature is that although the evening activity is delayed during warm days, the morning peak is advanced (e.g., see Figs. 1 and 3). Although this is almost certainly linked to the "desire" of minimizing daytime activity on hot days, how does the clock delay evening activity while advancing morning activity? Recent findings suggest some possibilities, such as differential phase adjustments in $\mathrm{M}$ and $\mathrm{E}$ clocks (Bachleitner et al. 2007). Moreover, the possibility that some pacemaker cells in the Drosophila brain might be preferentially responsive to temperature compared to light (Yoshii et al. 2005; Miyasako et al. 2007) suggests that a complex web of neural networks is involved in integrating photoperiodic and thermal cues to yield seasonably appropriate daily activity patterns. Finally, genome-wide expression studies indicate that information concerning ambient temperature and photoperiod has widespread and coordinate effects on circadian regulation of gene expression in Drosophila (Boothroyd et al. 2007). This work also indicated that an intron in tim is regulated by temperature, suggesting a broader role for thermosensitive splicing in clock responses to temperature. In addition, the recent demonstration that two different isoforms of tim have differential effects on photoperiod-induced ovarian diapause (Sandrelli et al. 2007; Tauber et al. 2007) further indicates that TIM is also a significant clock component in contributing to the seasonal adaptation of Drosophila physiology and behavior. Thus, the partnership between dPER and TIM is a key step for the circadian integration of thermal and photic information.

Of course, temperature and day length are highly associated in temperate climates, making it unsurprising that these two modalities are closely intertwined at the level of clock function. Nonetheless, as Pittendrigh noted "although photoperiod is a more reliable indicator of season and organisms have evolved sophisticated methods to measure its length, the physiological value of such a timing device is likely to be mainly directed at anticipating and thus preparing for changes in daily temperatures" (Pittendrigh and Takamura 1987). Recent work in Drosophila and Neurospora indicates that the thermosensitive splicing of central clock components is a common feature of how circadian clocks respond to temperature. Although other possibilities are likely, weak or nonconsensus splice signals that are more efficiently recognized at lower temperatures appear to be a common theme in generating temperature-dependent splicing of precursor mRNAs. On a broader perspective, RNA-RNA interactions with the ability to form a multitude of short- and long-range base-pair interactions varying in strength appear to be ideal targets for constructing intracellular molecular mechanisms that can be targeted by natural selection to generate highly calibrated thermal responses.

\section{ACKNOWLEDGMENTS}

We thank all the members in the Edery lab, past and present, for their many contributions to studies aimed at understanding the role of the clock in seasonal adaptation, especially John Majercak who initiated the work.

\section{REFERENCES}

Ainsworth J.R., Rossi L.M., and Murphy E.C., Jr. 1996. The Moloney murine sarcoma virus ts $1105^{\prime}$ splice site signal contributes to the regulation of splicing efficiency and thermosensitivity. J. Virol. 70: 6474.

Angelichio M.L., Beck J.A., Johansen H., and Ivey-Hoyle M. 1991. Comparison of several promoters and polyadenylation signals for use in heterologous gene expression in cultured Drosophila cells. Nucleic Acids Res. 19: 5037.

Aronson B.D., Johnson K.A., Loros J.J., and Dunlap J.C. 1994. Negative feedback defining a circadian clock: Autoregulation of the clock gene frequency. Science 263: 1578.

Bachleitner W., Kempinger L., Wulbeck C., Rieger D., and Helfrich-Förster C. 2007. Moonlight shifts the endogenous clock of Drosophila melanogaster. Proc. Natl. Acad. Sci. 104: 3538.

Boothroyd C.E., Wijnen H., Naef F., Saez L., and Young M.W. 2007. Integration of light and temperature in the regulation of circadian gene expression in Drosophila. PLoS Genet. 3: e54.

Bourgeois C.F., Lejeune F., and Stevenin J. 2004. Broad specificity of SR (serine/arginine) proteins in the regulation of alternative splicing of pre-messenger RNA. Prog. Nucleic Acid Res. Mol. Biol. 78: 37.

Brunner M. and Diernfellner A. 2006. How temperature affects the circadian clock of Neurospora crassa. Chronobiol. Int. 23: 81 .

Chen W.F., Majercak J., and Edery I. 2006. Clock-gated photic stimulation of timeless expression at cold temperatures and seasonal adaptation in Drosophila. J. Biol. Rhythms 21: 256.

Cheng Y., Gvakharia B., and Hardin P.E. 1998. Two alternatively spliced transcripts from the Drosophila period gene rescue rhythms having different molecular and behavioral characteristics. Mol. Cell. Biol. 18: 6505.

Cizdziel P.E., de Mars M., and Murphy E.C., Jr. 1988. Exploitation of a thermosensitive splicing event to study premRNA splicing in vivo. Mol. Cell. Biol. 8: 1558.

Collins B.H., Rosato E., and Kyriacou C.P. 2004. Seasonal behavior in Drosophila melanogaster requires the photoreceptors, the circadian clock, and phospholipase C. Proc. Natl. Acad. Sci. 101: 1945.

Colot H.V., Loros J.J., and Dunlap J.C. 2005. Temperature-modulated alternative splicing and promoter use in the Circadian clock gene frequency. Mol. Biol. Cell 16: 5563.

Diernfellner A.C., Schafmeier T., Merrow M.W., and Brunner M. 2005. Molecular mechanism of temperature sensing by the circadian clock of Neurospora crassa. Genes Dev. 19: 1968.

Dunlap J.C. 1996. Genetics and molecular analysis of circadian rhythms. Annu. Rev. Genet. 30: 579.

Edery I. 2000. Circadian rhythms in a nutshell. Physiol. Genomics 3: 59.

Edery I., Rutila J.E., and Rosbash M. 1994a. Phase shifting of the circadian clock by induction of the Drosophila period protein. Science 263: 237.

Edery I., Zwiebel L.J., Dembinska M.E., and Rosbash M. 1994b. Temporal phosphorylation of the Drosophila period protein. Proc. Natl. Acad. Sci. 91: 2260.

Gallego M. and Virshup D.M. 2007. Post-translational modifications regulate the ticking of the circadian clock. Nat. Rev. Mol. Cell Biol. 8: 139.

Garceau N.Y., Liu Y., Loros J.J., and Dunlap J.C. 1997. Alternative initiation of translation and time-specific phosphorylation yield multiple forms of the essential clock protein FREQUENCY. Cell 89: 469.

Glaser F.T. and Stanewsky R. 2005. Temperature synchronization of the Drosophila circadian clock. Curr. Biol. 15: 1352.

Grima B., Chelot E., Xia R., and Rouyer F. 2004. Morning and evening peaks of activity rely on different clock neurons of the Drosophila brain. Nature 431: 869.

Hamblen-Coyle M.J., Wheeler D.A., Rutila J.E., Rosbash M., and Hall J.C. 1992. Behavior of period-altered circadian rhythm mutants of Drosophila in light:dark cycles (Diptera: Drosophilidae). J. Insect Behav. 5: 417.

Hastings J.W., Rusak B., and Boulos Z. 1991. Circadian rhythms: The physiology of biological timing. In Neural and 
integrative animal physiology (ed. C.L. Prosser), p. 435. Wiley-Liss, New York.

Hastings M.L. and Krainer A.R. 2001. Pre-mRNA splicing in the new millennium. Curr. Opin. Cell Biol. 13: 302.

He Q., Cha J., He Q., Lee H.C., Yang Y., and Liu Y. 2006. CKI and CKII mediate the FREQUENCY-dependent phosphorylation of the WHITE COLLAR complex to close the Neurospora circadian negative feedback loop. Genes Dev. 20: 2552.

Heckrotte C. 1961. The effect of the environmental factors in the locomotor activity of the plains garter snake (Thamnophis radix radix). Anim. Behav. 10: 193.

Kim E.Y., Ko H.W., Yu W., Hardin P.E., and Edery I. 2007. A DOUBLETIME kinase binding domain on the Drosophila PERIOD protein is essential for its hyperphosphorylation, transcriptional repression, and circadian clock function. Mol. Cell. Biol. 27: 5014

Lakin-Thomas P.L., Brody S., and Cote G.G. 1991. Amplitude model for the effects of mutations and temperature on period and phase resetting of the Neurospora circadian oscillator. $J$. Biol. Rhythms 6: 281 .

Liu Y., Garceau N.Y., Loros J.J., and Dunlap J.C. 1997. Thermally regulated translational control of FRQ mediates aspects of temperature responses in the Neurospora circadian clock. Cell 89: 1 .

Liu Y., Merrow M., Loros J.J., and Dunlap J.C. 1998. How temperature changes reset a circadian oscillator. Science 281: 825.

Majercak J., Chen W.F., and Edery I. 2004. Splicing of the period gene 3 '-terminal intron is regulated by light, circadian clock factors, and phospholipase C. Mol. Cell. Biol. 24: 3359.

Majercak J., Sidote D., Hardin P.E., and Edery I. 1999. How a circadian clock adapts to seasonal decreases in temperature and day length. Neuron 24: 219.

Matsumoto A., Matsumoto N., Harui Y., Sakamoto M., and Tomioka K. 1998. Light and temperature cooperate to regulate the circadian locomotor rhythm of wild type and period mutants of Drosophila melanogaster. J. Insect Physiol. 44: 587.

Miyasako Y., Umezaki Y., and Tomioka K. 2007. Separate sets of cerebral clock neurons are responsible for light and temperature entrainment of Drosophila circadian locomotor rhythms. J. Biol. Rhythms 22: 115.

Nakajima M., Imai K., Ito H., Nishiwaki T., Murayama Y., Iwasaki H., Oyama T., and Kondo T. 2005. Reconstitution of circadian oscillation of cyanobacterial KaiC phosphorylation in vitro. Science 308: 414.

Pittendrigh C.S. and Daan S. 1976. A functional analysis of circadian pacemakers in nocturnal rodents. V. Pacemaker structure: A clock for all seasons. J. Comp. Physiol. A 106: 333.

Pittendrigh C.S. and Takamura T. 1987. Temperature dependence and evolutionary adjustment of critical night length in insect photoperiodism. Proc. Natl. Acad. Sci. 84: 7169.

Pittendrigh C.S., Kyner W.T., and Takamura T. 1991. The amplitude of circadian oscillations: Temperature dependence, latitudinal clines, and the photoperiodic time measurement. $J$. Biol. Rhythms 6: 299 .

Qiu J. and Hardin P.E. 1996. per mRNA cycling is locked to lights-off under photoperiodic conditions that support circadian feedback loop function. Mol. Cell. Biol. 16: 4182.

Rensing L. and Ruoff P. 2002. Temperature effect on entrainment, phase shifting, and amplitude of circadian clocks and its molecular bases. Chronobiol. Int. 19: 807.

Revel F.G., Herwig A., Garidou M.L., Dardente H., Menet J.S., Masson-Pevet M., Simonneaux V., Saboureau M., and Pevet P. 2007. The circadian clock stops ticking during deep hibernation in the European hamster. Proc. Natl. Acad. Sci. 104: 13816.
Rieger D., Stanewsky R., and Helfrich-Förster C. 2003. Cryptochrome, compound eyes, Hofbauer-Buchner eyelets, and ocelli play different roles in the entrainment and masking pathway of the locomotor activity rhythm in the fruit fly Drosophila melanogaster. J. Biol. Rhythms 18: 377.

Sandrelli F., Tauber E., Pegoraro M., Mazzotta G., Cisotto P., Landskron J., Stanewsky R., Piccin A., Rosato E., Zordan M., Costa R., and Kyriacou C.P. 2007. A molecular basis for natural selection at the timeless locus in Drosophila melanogaster. Science 316: 1898

Sawyer L.A., Hennessy J.M., Peixoto A.A., Rosato E., Parkinson H., Costa R., and Kyriacou C.P. 1997. Natural variation in a Drosophila clock gene and temperature compensation. Science 278: 2117.

Sayeed O. and Benzer S. 1996. Behavioral genetics of thermosensation and hygrosensation in Drosophila. Proc. Natl. Acad. Sci. 93: 6079.

Schafmeier T., Haase A., Kaldi K., Scholz J., Fuchs M., and Brunner M. 2005. Transcriptional feedback of Neurospora circadian clock gene by phosphorylation-dependent inactivation of its transcription factor. Cell 122: 235

Shafer O.T., Levine J.D., Truman J.W., and Hall J.C. 2004. Flies by night: Effects of changing day length on Drosophila's circadian clock. Curr. Biol. 14: 424.

Shannon K.W. and Guthrie C. 1991. Suppressors of a U4 snRNA mutation define a novel U6 snRNP protein with RNA-binding motifs. Genes Dev. 5: 773.

Sidote D., Majercak J., Parikh V., and Edery I. 1998. Differential effects of light and heat on the Drosophila circadian clock proteins PER and TIM. Mol. Cell. Biol. 18: 2004.

Stoleru D., Peng Y., Agosto J., and Rosbash M. 2004. Coupled oscillators control morning and evening locomotor behaviour of Drosophila. Nature 431: 862.

Stoleru D., Nawathean P., de la Paz Fernández M., Menet J.S. Ceriani M.F., and Rosbash M. 2007. The Drosophila circadian network is a seasonal timer. Cell 129: 207.

Sweeney B.M. and Hastings J.W. 1961. Effects of temperature upon diurnal rhythms. Cold Spring Harbor Symp. Quant. Biol. 25: 87.

Tauber E., Zordan M., Sandrelli F., Pegoraro M., Osterwalder N., Breda C., Daga A., Selmin A., Monger K., Benna C., Rosato E., Kyriacou C.P., and Costa R. 2007. Natural selection favors a newly derived timeless allele in Drosophila melanogaster. Science 316: 1895.

Touchman J.W., D'Souza I., Heckman C.A., Zhou R., Biggart N.W., and Murphy E.C., Jr. 1995. Branchpoint and polypyrimidine tract mutations mediating the loss and partial recovery of the Moloney murine sarcoma virus MuSVts110 thermosensitive splicing phenotype. J. Virol. 69: 7724.

Wheeler D.A., Hamblen-Coyle M.J., Dushay M.S., and Hall J.C. 1993. Behavior in light-dark cycles of Drosophila mutants that are arrhythmic, blind, or both. J. Biol. Rhythms 8: 67 .

Yoshii T., Sakamoto M., and Tomioka K. 2002. A temperaturedependent timing mechanism is involved in the circadian system that drives locomotor rhythms in the fruit fly Drosophila melanogaster. Zool. Sci. 19: 841.

Yoshii T., Heshiki Y., Ibuki-Ishibashi T., Matsumoto A., Tanimura T., and Tomioka K. 2005. Temperature cycles drive Drosophila circadian oscillation in constant light that otherwise induces behavioural arrhythmicity. Eur. J. Neurosci. 22: 1176.

Yu W., Zheng H., Houl J.H., Dauwalder B., and Hardin P.E. 2006. PER-dependent rhythms in CLK phosphorylation and E-box binding regulate circadian transcription. Genes Dev. 20: 723 . 


\section{$\overbrace{\text { CSH' }}^{\infty}$ Cold Spring Harbor Symposia SYMPOSIA}

\section{Thermosensitive Splicing of a Clock Gene and Seasonal Adaptation}

W.-F. Chen, K. H. Low, C. Lim, et al.

Cold Spring Harb Symp Quant Biol 2007 72: 599-606

Access the most recent version at doi:10.1101/sqb.2007.72.021

References This article cites 57 articles, 26 of which can be accessed free at: http://symposium.cshlp.org/content/72/599.full.html\#ref-list-1

\section{License} Email Alerting $\begin{aligned} & \text { Receive free email alerts when new articles cite this article - sign up in the box at the } \\ & \text { Service }\end{aligned}$ top right corner of the article or click here. 